\title{
The biorthogonal wavelets that are redundant-free and nearly shift-insensitive
}

\author{
Hongli Shi ${ }^{*}$ and Shuqian Luo
}

\begin{abstract}
It is well known that discrete wavelet transform (DWT) is sensitive to shift, which means a slight shift of feature in the original signal may cause unpredictable changes in the analysis subbands. Some modified versions of DWT can reduce the shift sensitivity, however, they are all redundant. In this article, we shows the shift sensitivity is caused by the aliasing terms formed in the downsampling operation during analysis process. A novel scheme for the design of wavelet is proposed to reduce the effect of aliasing terms as much as possible in the general framework of DWT. A few of biorthogonal wavelets have been designed and applied in the simulation examples. The results of examples demonstrate the efficiency of the designed wavelets in the term of shift insensitivity and nonredundancy.
\end{abstract}

Keywords: Biorthogonal wavelet, DWT, Shift sensitivity

\section{Introduction}

Discrete wavelet transform (DWT) has been applied in many fields as a tool of signal processing, e.g., signal denoising, feature extraction, pattern recognition and image registration [1-3]. However, DWT is shift-sensitive [4]. A slight shift of feature in the original signals or images may generate unpredictable changes in its DWT analysis subbands. For example, for level- $d$ low-low (LL) subbands of a two-dimensional figure, only the features that consist of more than $2^{2 d}$ pixels in the original images can be insensitive to shift in image registration [5]. Some new wavelets and modified calculation frameworks of DWT have been presented to reduce the shift sensitivity. However, all these DWTs become redundant, i.e., they are no longer critically-sampled [6-10]. For example, Kingsbury's dual tree complex wavelet transform (DTCWT) and Selesnick's double-density wavelet transform (DDWT) are all redundant. A pair of filter banks is employed in Kingsbury's DTCWT, which leads to a constant redundancy rate of $2: 1$ for 1 -D signals and $2^{m}: 1$ for m-dimensional signals. The other modified DWTs, such as DDWT, also cannot be critically-sampled. The lack of directionality is other main drawback of DWTs. Many modified transformations have been presented to improve directional

*Correspondence: shl@ccmu.edu.cn

College of Biomedical Engineering, Capital Medical University, Beijing 100069, China selectivity, such as curvelet and contourlet transformations [11-13]. These multiresolution representations are much more redundant.

The shift sensitivity of DWT can be expressed in many ways. In this article, it is expressed in the frequency domain by the aliasing terms formed in downsampling operation during analysis process (see Figure 1). According to this point, a new method for the design of wavelet is proposed to reduce the effect of aliasing terms. Some extra requirements are introduced besides the basic requirements (i.e., the perfect reconstruction requirement) on wavelets. In the design, we focus on the design of biorthogonal wavelets because they are symmetrical (or linear-phase) and have been used in many fields. The general calculation framework (Figure 1) is remained to keep DWT non-redundant. A few of biorthogonal wavelets have been designed, and applied in some simulation examples. The results of the design examples illustrate the shift sensitivity has been reduced efficiently.

\section{The shift sensitivity of DWT}

Consider the calculation framework of DWT using biorthogonal wavelet in Figure 1, in which $H_{0}$ and $\widetilde{\widetilde{H}}_{0}(z)$ denote the analysis and synthesis filters in the low-pass branch. $H_{0}$ and $\widetilde{H}_{0}(z)$ can be designed to be symmetrical to ensure linear-phase. $\widetilde{H}_{0}(z)$ denotes the complex conjugate of $\widetilde{H}_{0}(z) . H_{1}$ and $\widetilde{H}_{1}(z)$ are the corresponding filters in

\section{Springer}

(c) 2012 Shi and Luo; licensee Springer. This is an Open Access article distributed under the terms of the Creative Commons Attribution License (http://creativecommons.org/licenses/by/2.0), which permits unrestricted use, distribution, and reproduction in any medium, provided the original work is properly cited. 


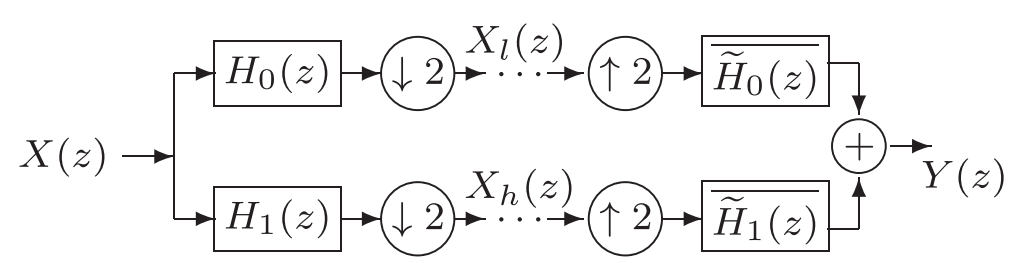

Figure 1 DWT framework: analysis and synthesis using the biorthogonal wavelet.

the high-pass branch. Let $X(z)$ denote the original signal. The analysis subbands become

$$
\begin{aligned}
X_{l}(z)= & {\left[X(z) H_{0}(z)\right] \downarrow 2=\frac{1}{2} X\left(z^{\frac{1}{2}}\right) H_{0}\left(z^{\frac{1}{2}}\right) } \\
& +\frac{1}{2} X\left(-z^{\frac{1}{2}}\right) H_{0}\left(-z^{\frac{1}{2}}\right) \\
X_{h}(z)=[ & \left.X(z) H_{1}(z)\right] \downarrow 2=\frac{1}{2} X\left(z^{\frac{1}{2}}\right) H_{1}\left(z^{\frac{1}{2}}\right) \\
& +\frac{1}{2} X\left(-z^{\frac{1}{2}}\right) H_{1}\left(-z^{\frac{1}{2}}\right)
\end{aligned}
$$

where “ $\downarrow 2$ " denotes the downsampling operation by the factor of " 2 ". The synthesis signal $Y(z)$ becomes

$$
\begin{aligned}
Y(z)= & \overline{\widetilde{H}_{0}(z)}\left[X_{l}(z) \uparrow 2\right]+\overline{\widetilde{H}_{1}(z)}\left[X_{h}(z) \uparrow 2\right] \\
= & \frac{1}{2} X(z)\left[\overline{\widetilde{H}_{0}(z)} H_{0}(z)+\overline{\widetilde{H}_{1}(z)} H_{1}(z)\right] \\
& +\frac{1}{2} X(-z)\left[\overline{\widetilde{H}_{0}(z)} H_{0}(-z)+\overline{\widetilde{H}_{1}(z)} H_{1}(-z)\right]
\end{aligned}
$$

where " $\uparrow 2$ " denotes the upsampling process by the factor of " 2 ". The no-aliasing condition and no-distortion condition require

$$
\begin{aligned}
& \overline{\widetilde{H}_{0}(z)} H_{0}(-z)+\overline{\widetilde{H}_{1}(z)} H_{1}(-z)=0 . \\
& \overline{\widetilde{H}_{0}(z)} H_{0}(z)+\overline{\widetilde{H}_{1}(z)} H_{1}(z)=2 z^{k}, k \in \mathbb{Z} .
\end{aligned}
$$

The no-aliasing and no-distortion conditions ensure that the aliasing terms in Equation (1), $\frac{1}{2} X\left(-z^{\frac{1}{2}}\right) H_{0}\left(-z^{\frac{1}{2}}\right)$ and $\frac{1}{2} X\left(-z^{\frac{1}{2}}\right) H_{1}\left(-z^{\frac{1}{2}}\right)$, will be eliminated in synthesis process, and the original signal can be perfectly reconstructed. However, the wavelet representation in the analysis output, $X_{l}(z)$ and $X_{h}(z)$, become shift-sensitive because of the aliasing terms. The shift sensitivity can be shown in many ways. Here, it is shown in the following way. Suppose $X(z)$ is delayed by one sample and denoted as $X^{\prime}(z)$, i.e., $X^{\prime}(z)=z^{-1} X(z)$, the low-pass output $X_{l}^{\prime}(z)$ becomes

$$
\begin{aligned}
X_{l}^{\prime}(z) & =\left[z^{-1} X(z) H_{0}(z)\right] \downarrow 2 \\
& =\frac{1}{2} z^{-\frac{1}{2}}\left[X\left(z^{\frac{1}{2}}\right) H_{0}\left(z^{\frac{1}{2}}\right)-X\left(-z^{\frac{1}{2}}\right) H_{0}\left(-z^{\frac{1}{2}}\right)\right] \\
& \neq z^{-\frac{1}{2}}\left[X_{l}(z)\right]
\end{aligned}
$$

A shift-invariant transform $\mathcal{F}(\cdot)$ requires that $\mathcal{F}\left(z^{k_{1}} X[z]\right)=z^{k_{2}} \mathcal{F}(X[z])$, where $k_{1}, k_{2} \in \mathbb{R}$ are the constants. Therefore, the aliasing terms $\frac{1}{2} X\left(-z^{\frac{1}{2}}\right) H_{0}\left(-z^{\frac{1}{2}}\right)$ brings out the shift sensitivity in the low-pass analysis output. The shift sensitivity is very troublesome in many applications. For example, the original signal $x(n)$ in Figure 2a consists of five pulses (the pulse width is 2-pixel) at different positions (the period is 101-pixels) with the same magnitudes. It equals to a pulse and its shifted versions. The low-pass output of first level DWT are shown in Figure 2b using biorthogonal wavelet "bior3.9" (the analysis and synthesis filters are 20- and 4-tap, respectively). It shows analysis outputs of these pulses becomes quite different. Generally, the low-pass subbands are more insensitive to shift than the highpass subbands. Even though for the low-pass subbands, the shift sensitivity is still unacceptable in some applications. With wavelet analysis proceeding, the effect of shift sensitivity may becomes more and more serious. In the following section, we will propose a new scheme to reduce the effect of aliasing terms, and thus reduce shift sensitivity.

\section{The nearly shift-invariant and critically-sampled DWT}

In order to avoid redundancy, the framework of general DWT, Figure 1, is remained in the proposed DWT except the wavelet filters are designed to satisfy some extra requirements. 


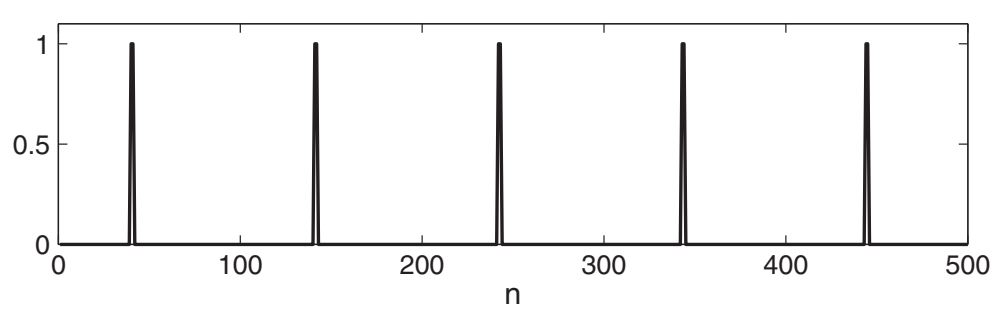

(a)

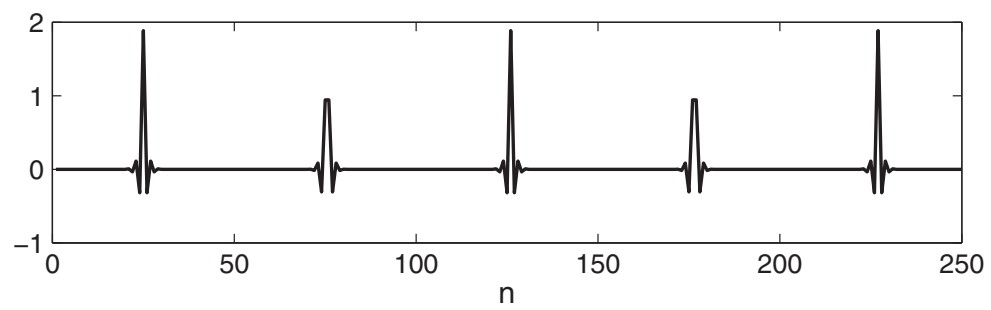

(b)

Figure 2 The original signal and the analysis output of first level DWT using wavelet "bior3.9". (a) The original signal, (b) the low-pass subband.

\section{The extra requirements on wavelets}

In order to reduce the effect of aliasing terms in the lowpass analysis output, $H_{0}(z)$ in Figure 1 is designed to be expressed as (4) and satisfy (5).

$$
H_{0}(z)=P(z)\left[F\left(z^{2}\right)+z^{-1} G\left(z^{2}\right)\right]
$$

where $P(z)$ is a low-pass FIR filter; $F(\cdot)$ and $G(\cdot)$ denotes two FIR filters satisfy

$$
F(z) \approx z^{-1 / 2} G(z)
$$

Table 1 The filter bank $(Q(z)$ and $\widetilde{Q}(z)$ are 9- and 3-tap, $k=11, \tilde{k}=1$ )

\begin{tabular}{cccc}
\hline $\boldsymbol{n}$ & $\boldsymbol{F}$ & $\boldsymbol{n}$ & $\boldsymbol{G}$ \\
\hline-12 & -1.620457579834970 & -22 & 0.183914612820184 \\
01 & -14.856833358190139 & -11 & 6.521032167975675 \\
& & 0 & 20.173189134221989 \\
$\boldsymbol{n}$ & $\boldsymbol{H}_{\mathbf{0}}$ & $\boldsymbol{n}$ & $\tilde{\boldsymbol{H}}_{\mathbf{0}}$ \\
-109 & 0.000359208228164 & -12 & -0.334521746568079 \\
-98 & 0.000786334299193 & 01 & 0.732293701891982 \\
-87 & -0.002321674814646 & & \\
-76 & -0.003720186105568 & & \\
-65 & 0.017033423809928 & & \\
-54 & 0.031465370920245 & & \\
-43 & -0.020504827773706 & & \\
-32 & 0.006960043873026 & & \\
-21 & 0.373526016710723 & & \\
-10 & 0.853417930379625 & & \\
\hline
\end{tabular}

Consequently,

$$
\begin{aligned}
X_{l}(z)= & {\left[X(z) H_{0}(z)\right] \downarrow 2 } \\
= & \frac{1}{2} X\left(z^{\frac{1}{2}}\right) P\left(z^{\frac{1}{2}}\right)\left[F(z)+z^{-\frac{1}{2}} G(z)\right] \\
& +\frac{1}{2} X\left(-z^{\frac{1}{2}}\right) P\left(-z^{\frac{1}{2}}\right)\left[F(z)-z^{-\frac{1}{2}} G(z)\right] \\
\approx & X\left(z^{\frac{1}{2}}\right) P\left(z^{\frac{1}{2}}\right) F(z)
\end{aligned}
$$

Table 2 The filter bank $(Q(z)$ and $\widetilde{Q}(z)$ are 19- and 3-tap, $k=\tilde{k}=1$ )

\begin{tabular}{cccc}
\hline $\boldsymbol{n}$ & $\boldsymbol{F}$ & $\boldsymbol{n}$ & $\boldsymbol{G}$ \\
\hline-45 & 0.036875746448608 & -44 & 0.011220065157315 \\
-34 & -0.023135612111302 & -33 & 0.033448583455217 \\
-23 & 0.117860494348029 & -22 & 0.104853324526458 \\
-12 & 0.087646010428631 & -11 & 0.302593640841847 \\
01 & 0.710918453811750 & 0 & 0.927449000805610 \\
$\boldsymbol{n}$ & $\boldsymbol{H}_{\mathbf{0}}$ & $\boldsymbol{n}$ & $\tilde{\boldsymbol{H}}_{\mathbf{0}}$ \\
-109 & 0.036875746448607 & -21 & -0.445094807808344 \\
-98 & 0.048095811605923 & -10 & 0.580522378115357 \\
-87 & -0.011915546953987 & & \\
-76 & 0.010312971343915 & & \\
-65 & 0.151309077803246 & & \\
-54 & 0.222713818874487 & & \\
-43 & 0.192499334955090 & & \\
-32 & 0.390239651270478 & & \\
-21 & 1.013512094653598 & & \\
-10 & 1.638367454617361 & & \\
\hline
\end{tabular}



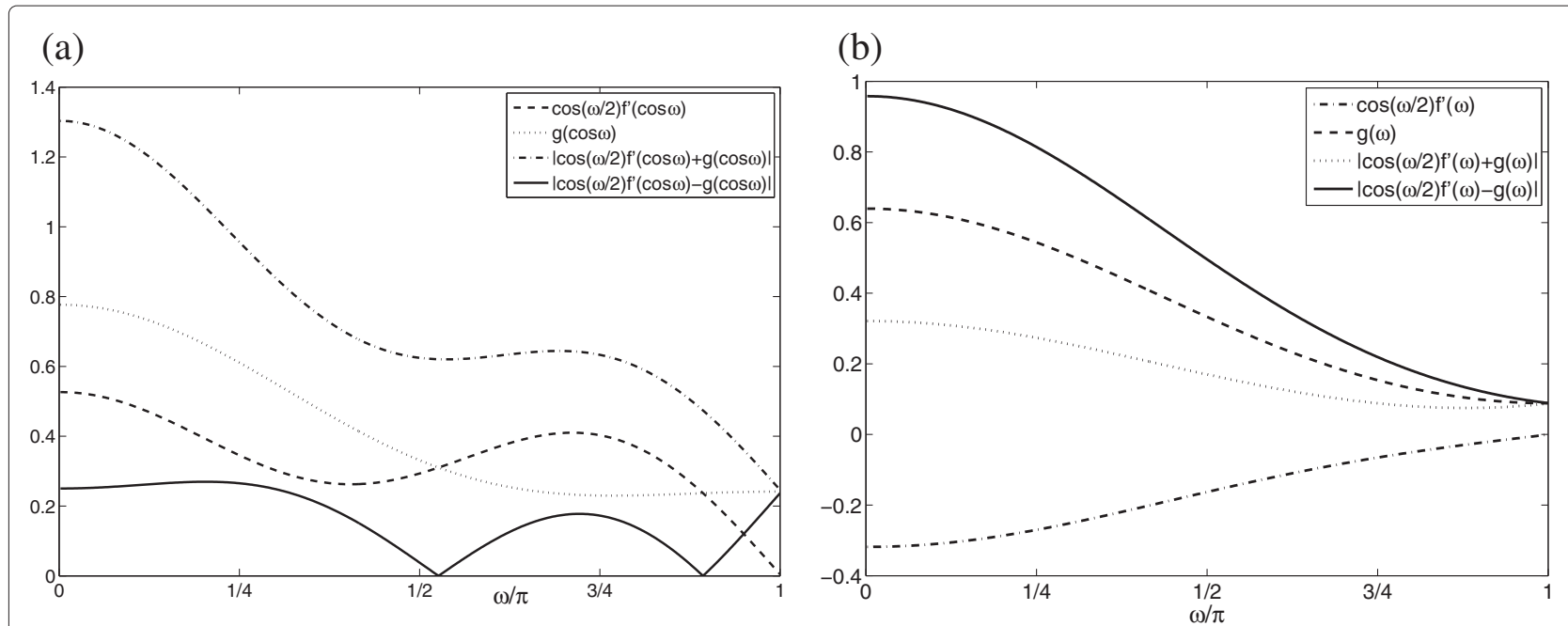

Figure 3 The magnitude responds of filters $F(z)$ and $G(z)$ for the filters in Table 2 and "bior3.9". (a) $\cos (\omega / 2) f^{\prime}(\cos \omega), g(\cos \omega)$, $\left|\cos (\omega / 2) f^{\prime}(\cos \omega)+g(\cos \omega)\right|$ and $\left|\cos (\omega / 2) f^{\prime}(\cos \omega)-g(\cos \omega)\right|$ for the filters in Table 2, (b) the corresponding curves for wavelet "bior3.9".

$$
\begin{aligned}
X_{l}^{\prime}(z)= & \frac{1}{2} z^{-\frac{1}{2}}\left[X\left(z^{\frac{1}{2}}\right) P\left(z^{\frac{1}{2}}\right)\left(F(z)+z^{-\frac{1}{2}} G(z)\right)\right. \\
& \left.-X\left(-z^{\frac{1}{2}}\right) P\left(-z^{\frac{1}{2}}\right)\left(F(z)-z^{-\frac{1}{2}} G(z)\right)\right] \\
\approx & z^{-\frac{1}{2}} X\left(z^{\frac{1}{2}}\right) P\left(z^{\frac{1}{2}}\right) F(z) \approx z^{-\frac{1}{2}} X_{l}(z)
\end{aligned}
$$

Namely, the effect of aliasing terms in the low-pass subband has been approximately removed. The design scheme of wavelets that satisfy these extra requirements will be given in the next. First of all, it is necessary to review the design of biorthogonal wavelet.

\section{The design of biorthogonal wavelet}

Consider $H_{0}(z)$ and $H_{0}(z)$ in Figure 1. In order to obtain smooth wavelet bases, it is always imposed certain numbers of zeros at $z=-1$ for $H_{0}(z)$ and $\widetilde{H}_{0}(z)$, i.e., the filter has certain numbers of vanishing moments [14]. Suppose $H_{0}(z)$ is expressed as $H_{0}(z)=\frac{1}{\sqrt{2}}\left(\frac{1+z^{-1}}{2}\right)^{k} Q(z)$, where $k \in \mathbb{Z}^{+}, Q(z)$ is an odd symmetrical filters of $(2 l+1)$ $\operatorname{tap}$ (if $Q(z)$ is a symmetric filter of $(2 l+2)$-tap, it can be expressed as $Q(z)=\frac{1+z^{-1}}{2} Q^{\prime}(z)$ with $Q^{\prime}(z)$ a symmetric filter of $(2 l+1)$-tap). Similarly, $\widetilde{H}_{0}(z)$ is expressed as $\widetilde{H}_{0}(z)=\frac{1}{\sqrt{2}}\left(\frac{1+z^{-1}}{2}\right)^{k} \widetilde{Q}(z)$, where $\widetilde{Q}(z)$ is is a symmetric

(a)

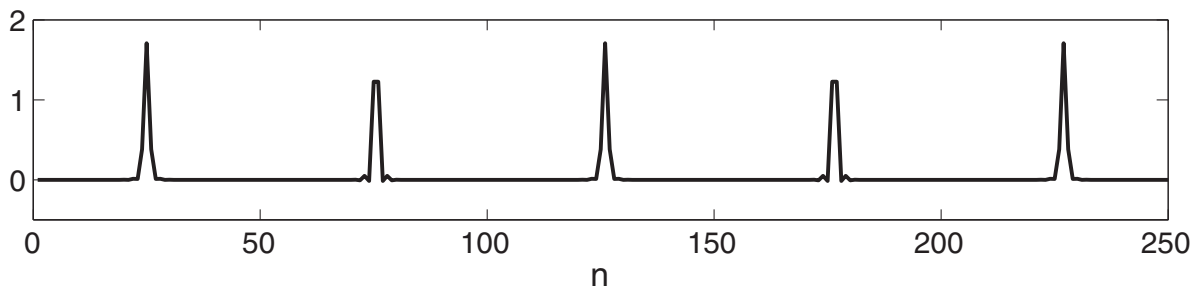

(b)

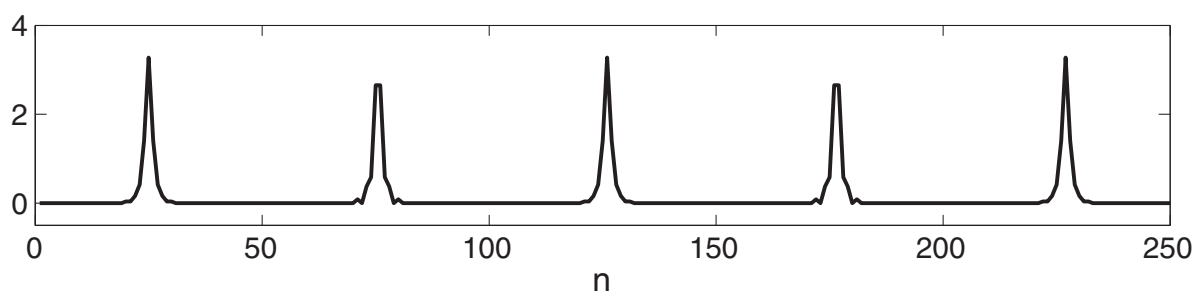

Figure 4 The low-pass subband of first level wavelet analysis using the designed wavelets. (a) Using the filters in Table 1, (b) Using the filters in Table. 
filter of $(2 \tilde{l}+1)$-tap, $\tilde{k} \in \mathbb{Z}^{+}$. By introducing a suitable integer translation, $Q(z)$ and $\widetilde{Q}(z)$ can be expressed as $Q(z)=q(\cos \omega)$ and $\widetilde{Q}(z)=\tilde{q}(\cos \omega)$, where $q(\cdot)$ and $\tilde{q}(\cdot)$ are two polynomials of real coefficients. According to the solution in [15], we have

$$
Q(z) \widetilde{Q}(z)=q(\cos \omega) \overline{\tilde{q}(\cos \omega)}=P_{N}(y)+y^{N} R(0.5-y)
$$

where

$$
P_{N}(y)=\sum_{k=0}^{N-1}\left(\begin{array}{c}
N-1+k \\
k
\end{array}\right) y^{k}
$$

$y=\sin ^{2}(\omega / 2), N=(k+\tilde{k}) / 2$ (it means the vanishing moments of $H_{0}(z)$ and $\widetilde{H}_{0}(z)$ must be either both odd or both even); $R(\cdot)$ is an odd polynomial, which is chosen such that $Q(z) \widetilde{Q}(z) \geq 0$ for all $\omega \in[0, \pi]$.

We can obtain $l+\tilde{l}+1$ constraints about the coefficients of $Q(z), \widetilde{Q}(z)$ and $R(\cdot)$ (Though there are $2 l+2 \tilde{l}+2$ equations on $Q(z)$ and $\widetilde{Q}(z)$, only $l+\tilde{l}+1$ of them are independent because of symmetry). It has been shown even when $R(\cdot)=0, Q(z)$ and $\widetilde{Q}(z)$ may be not unique for a identical $Q(z) \widetilde{\widetilde{Q}}(z)$. Furthermore, when $R(\cdot) \neq 0$, there are more choices in designing $Q(z)$ and $\widetilde{Q}(z)$, so are $H_{0}(z)$ and $\widetilde{H}_{0}(z)$. Therefore, it is possible to construct biorthogonal wavelet filters that satisfy the PR requirement and approximately satisfy (4) and (5). The design scheme is presented in the following section.

\section{The design of biorthogonal wavelets for the proposed DWT}

First, consider the PR requirement. From the requirements (4), we have $H_{0}(z)=P(z)\left[F\left(z^{2}\right)+z^{-1} G\left(z^{2}\right)\right]$. On the other hand, the general expression of $H_{0}(z)$ is $H_{0}(z)=\frac{1}{\sqrt{2}}\left(1+z^{-1}\right)^{k} Q(z)$. Thus, let $P(z)=\frac{1}{\sqrt{2}}(1+$ $\left.z^{-1}\right)^{k}$ (it is a low-pass filter), $Q(z)=F\left(z^{2}\right)+z^{-1} G\left(z^{2}\right)$. $F\left(z^{2}\right)+z^{-1} G\left(z^{2}\right)$ can be constructed in the following way. Suppose $F(z)$ denotes a symmetrical filter of $2 l$-tap $f, f=\left[f_{l-1}, \ldots, f_{0}, f_{0}, \ldots, f_{l-1}\right], G(z)$ denotes a $(2 l-1)$ tap symmetrical filter $g=\left[g_{l-1}, \ldots, g_{0}, \ldots, g_{l-1}\right]$, then $\left[f_{l-1}, g_{l-1}, \ldots, f_{0}, g_{0}, f_{0}, \ldots, g_{l-1}, f_{l-1}\right]$ becomes a symmetrical filter of $(4 l-1)$-tap. This filter can be expressed as $F\left(z^{2}\right)+z^{-1} G\left(z^{2}\right)$, where $F(z)$ and $G(z)$ are $z$-transform of $f$ and $g$, respectively. Let $\widetilde{Q}(z)$ be a symmetrical filter of $(2 m-1)$-tap. After a suitable integer translation and some manipulations, we have

$Q(z) \overline{\widetilde{Q}(z)}=\left(F\left(z^{2}\right)+z^{-1} G\left(z^{2}\right)\right) \overline{\widetilde{Q}(z)}=\sum_{n=0}^{2 l+m-2} a_{n}\left(z+z^{-1}\right)^{n}$

where $a_{n}, n=0, \ldots, 2 l+m-2$, are the real coefficients depending on $f$ and $g$. On the other hand, consider $y=$ $\sin ^{2} \frac{\omega}{2}=\frac{1}{2}-\frac{z^{-1}+z}{4}$, the right side of (7) can be simplified as following

$$
Q(z) \overline{\widetilde{Q}(z)}=\sum_{n=0}^{2 l+m-2} b_{n}\left(z+z^{-1}\right)^{n}
$$

Therefore, the PR requirement can be expressed as

$$
a_{i}=b_{i}, \quad i=0,1, \ldots, 2 l+m-2 .
$$

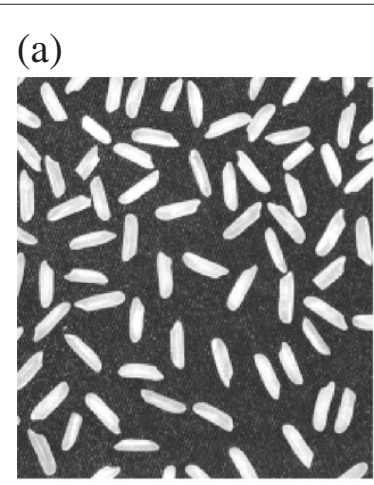

(c)

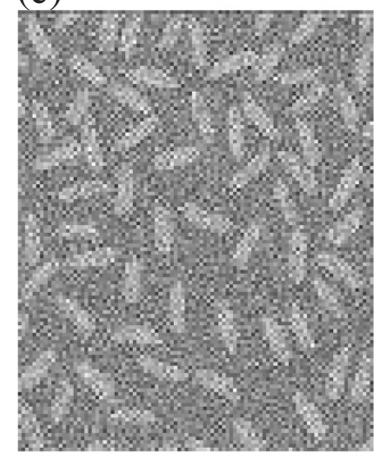

(e)

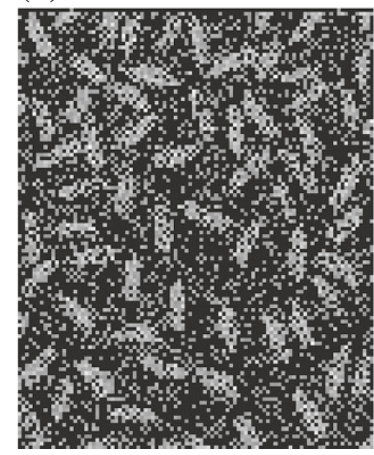

(b)

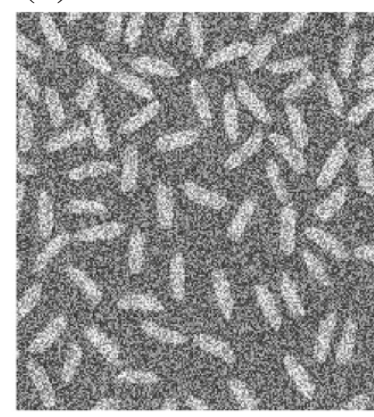

(d)

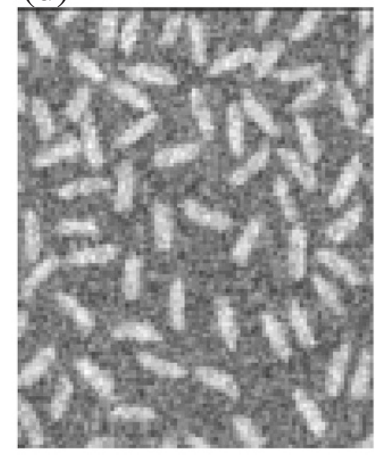

(f)

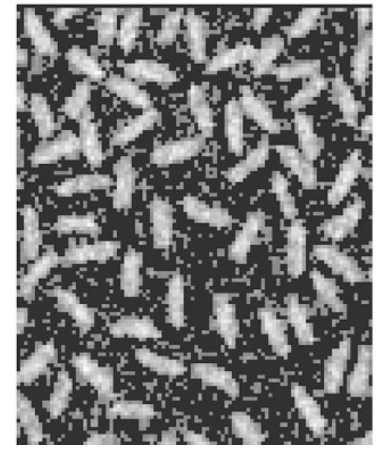

Figure 5 The original image, the noisy image, and the LL subbands of first level analysis using different wavelets. (a) The original image $(238 \times 211$ pixels), (b) the noisy image, (c) the LL subband (119 × 96 pixels) decomposed using "bior3.9", (d) the LL subband decomposed using the wavelet in Table 1, (e) the pixels in (c) whose intensities are smaller than $0.4 I_{\max }$ are set as $I_{\min }$, (f) the pixels (d) whose intensities are smaller than $0.4 I_{\max }$ are set as $I_{\min }$, where $I_{\max }$ and $I_{\min }$ are the maximum and minimum of each image, respectively. 
Second, consider the extra requirements (5). Since it is even symmetrical, $f$ can be factorized as $f=$ $f^{\prime} \otimes[1 / 2,1 / 2], f^{\prime}=\left[f_{l-1}^{\prime}, \ldots, f_{0}^{\prime}, \ldots, f_{l-1}^{\prime}\right]$ is a symmetrical filter, where $\otimes$ denotes the convolution operator. It means $F(z)$ can be expressed as $F(z)=z^{-1 / 2} \cos (\omega / 2) F^{\prime}(z)$. After a suitable translation, $F^{\prime}(z)$ can be simplified as $f^{\prime}(\cos \omega)$, where $f^{\prime}(\cdot)$ a real coefficient polynomial. Similarly, $g=\left[q_{l-1}, \ldots, q_{0}, \ldots, q_{l-1}\right]$ can be expressed as a real coefficient polynomial $g(\cos \omega)$. Therefore, if

$$
\cos (\omega / 2) f^{\prime}(\cos \omega) \approx g(\cos \omega)
$$

then

$$
F(z) \approx z^{-1 / 2} G(z)
$$

Therefore, the design can be simplified as a constrained optimization problem. The PR requirement becomes the constrained conditions certainly. The objective function can be selected as the integral of the square difference between $\left|\cos (\omega / 2) f^{\prime}(\cos \omega)\right|$ and $|g(\cos \omega)|$ for all $\omega \in$ $[0, \pi]$. It results

$$
\begin{gathered}
\min \int_{-\pi}^{\pi}(\cos (\omega / 2) f(\cos \omega)-g(\cos \omega))^{2} d \omega \\
\text { s.t. } \quad a_{i}=b_{i}, i=0,1, \ldots, N .
\end{gathered}
$$

\section{The design and application examples}

In this section, two design examples are presented to illustrate the design process, and three simulation examples are employed to demonstrate the performances of designed wavelets in the applications.

\section{The design examples}

Example 1. Two biorthogonal that approximately satisfy (5) are designed. In the first bank, $F$ and $G$ are 5and 4-tap, respectively, $\left(Q(z)=F(z)+z^{-1} G(z)\right.$ becomes 9-tap); $\widetilde{Q}(z)$ is 3-tap; $k=11, \tilde{k}=1$ (it means $R(\cdot)=$ 0 in Equation (7)). $H_{0}$ and $\widetilde{H}_{0}$ become 20- and 4-tap, respectively, which are identical with "bior3.9" wavelet filter banks in structure. The filter coefficients obtained are given in Table 1. It can be verified that $H_{0}$ and $\widetilde{H}_{0}$ satisfies the PR requirement (3) (Let $H_{1}(z)=-{\widetilde{H_{0}}}_{0}(-z)$ and $\left.\widetilde{H}_{1}(z)=-\overline{H_{0}(-z)}\right)$. In the second filter bank, $H_{0}$ and $\widetilde{H}_{0}$ are also 20 - and 4-tap, however, we choose $R(\cdot) \neq$ 0 to improve the approximation performance. The filter coefficients are shown in Table 2.

Equation (6) shows that if $\left[F(z)-z^{-\frac{1}{2}} G(z)\right] \approx 0$, $X_{l}(z) \approx X\left(z^{\frac{1}{2}}\right) P\left(z^{\frac{1}{2}}\right) F(z)$. Therefore, $\left[F(z)-z^{-\frac{1}{2}} G(z)\right]$ can be treated as the criteria to evaluate the design results. Consider $F(z)=z^{-1 / 2} \cos (\omega / 2) f^{\prime}(\cos \omega), G(z)=$ $g(\cos \omega), \angle F(z)-\angle z^{-\frac{1}{2}} G(z)=0$, i.e., the requirement of phase offset is satisfied perfectly. The magnitude responds, $\quad \cos (\omega / 2) f^{\prime}(\cos \omega), \quad g(\cos \omega), \quad \mid \cos (\omega / 2) f^{\prime}$ $(\cos \omega)+g(\cos \omega) \mid$ and $\left|\cos (\omega / 2) f^{\prime}(\cos \omega)-g(\cos \omega)\right|$ of Table 2 are depicted in Figure 3a. The corresponding magnitude responds of wavelet "bior3.9" is depicted in Figure $3 \mathrm{~b}$. It shows the designed wavelet has a better approximation performance to (6) than "bior3.9".

\section{The application examples}

Example 2. In a 1D example, the original signal is identical to that in Figure 2a. The filter banks in Tables 1 and 2 are used in wavelet analysis. The low-pass analysis outputs are shown in Figure 4a,b. The ratio of the minimum to the maximum of the five pules in Figure $2 b$ is 0.501 , while the corresponding ratios in Figure 4a,b is 0.719 and 0.809. It illustrates the effect of shift sensitivity has been reduced using the designed wavelets.

Example 3. In a 2D example, the original image is a noise-free image shown in Figure $5 \mathrm{a}$, in where the intensities of pixels range from 0 to 255 . It is corrupted with an additive noise uniformly distributed between 0 and 500, which is shown in Figure 5b. The noisy figure is decomposed using the wavelet "bior3.9" and the wavelet in Table 1. The low-low (LL) subbands of first level analysis are shown in Figure 5c,d.

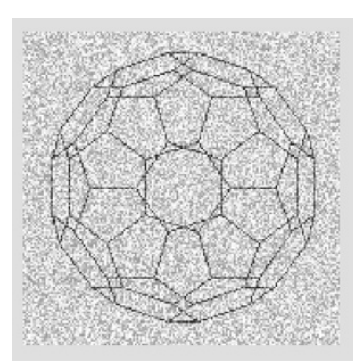

(a)

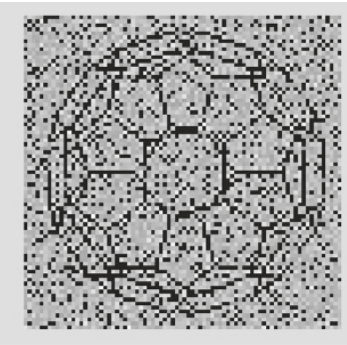

(b)

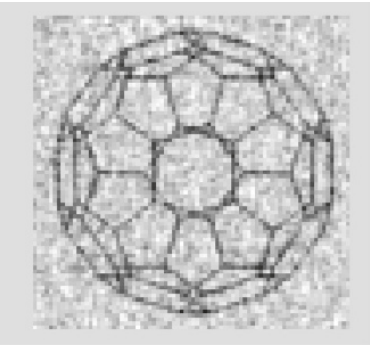

(c)

Figure $\mathbf{6}$ LL subbands of first level analysis using different waThe original image and thevelets. (a) The original image $(160 \times 160$ pixels), (b) the LL subband $(80 \times 80$ pixels) decomposed using "bior3.9", whose pixel intensities are smaller than $0.55 /$ max are set as $/ \mathrm{min}$, (c) the LL subband $(80 \times 80$ pixels) decomposed using the wavelet in Table 1, whose pixel intensities are smaller than $0.55 /$ max are set as $/ \mathrm{min}$. 
In order to show the intensity difference after 2D wavelet analysis, the pixels whose intensities are smaller than a certain percent of $I_{\max }$ have been set as $I_{\min }$, where $I_{\max }$ and $I_{\min }$ are the maximum and minimum of the LL subband coefficients, respectively. This operation is similar to the feature extraction according to the pixel intensities automatically. When the pixels in Figure $5 \mathrm{c}, \mathrm{d}$ whose intensities are smaller than $0.4 I_{\max }$ are set as $I_{\min }$, Figure 5c,d become Figure 5e,f. It also illustrates the effect of shift sensitivity has been reduced efficiently using the designed wavelets.

Example 4. In this example, the original image is also a corrupted image shown in Figure 6a. The figure is decomposed using the wavelet "bior3.9" and the wavelet in Table 1. The operations that are similar with Example 3 are performed to the two LL subbands: the pixels whose intensities are smaller than $0.55 I_{\max }$ are set as $I_{\min }$. Figure $6 \mathrm{~b}$ shows the LL subband decomposed using the wavelet "bior3.9", and (c) shows the one decomposed using the wavelet "bior3.9". It also illustrates the effect of shift sensitivity has been reduced efficiently using the designed wavelets.

\section{Conclusion}

For the general wavelets, the aliasing terms formed in analysis process of DWT can be eliminated in the synthesis process by the anti-aliasing properties of filter banks. However, the aliasing terms remain in the analysis outputs. In this article, it shows the aliasing terms cause the shift sensitivity of DWT. A novel scheme is proposed to reduce the effect of aliasing terms. Some extra requirements on the design of wavelets are proposed. The design scheme are presented and two biorthogonal wavelets that approximately satisfy the extra requirements have been designed. The shift sensitivity of the wavelet analysis has been reduced effectively by the new wavelets, which is very favorable for many signal processing applications, such as image registration, feature extraction and pattern recognition (the process is usually achieved using the analysis outputs rather than the synthesis results in these cases). The other superiority of the proposed wavelet analysis is that the wavelet representation remains critically-sampled and does not bring out any redundancy.

\section{Competing interest}

The authors declare that they have no competing interests.

\section{References}

1. A Abbate, CM DeCusatis, PK Das, Wavelets and Subbands: Fundamentals and Applications. (Birkhäuser, Boston, 2002), pp. 103-109

2. I Zavorin, JL Moigne, Use of multiresolution wavelet feature pyramids for automatic registration of multisensor imagery. IEEE Trans. Image Process 14(6), 770-782 (2005)

3. G De Grandi, J-S Lee, DL Schuler, Target detection and texture segmentation in polarimetric SAR images using a wavelet frame: theoretical aspects. IEEE Trans. Geosci. Remote Sens. 45(11), 3437-3453 (2007)

4. EP Simoncelli, WT Freeman, EH Adelson, DJ Heeger, Shiftable Multiscale Transforms. IEEE Trans. Inf. Theory. 38(2), 587-607 (1992)

5. HS Stone, JL Moigne, M McGuire, The translation sensitivity of wavelet-based registration IEEE Trans. Pattern Anal. Mach. Intell. 21, 1074-1081 (1999)

6. BN Kingsbury, Image processing with complex wavelets. Phil. Trans. Roy. Soc. Lond. 357(1760), 2543-2560 2543-2560 (1999)

7. FCA Fernandes, RLC van Spaendonck, CS Burrus, A New Framework for complex wavelet transforms. IEEE Trans. Signal Process. 51(7), 1825-1837 (2003)

8. RA Gopinath, The phaselet transform-an integral redundancy nearly shift-invariant wavelet transform. IEEE Trans. Signal Process. 51(7), 1792-1805 (2003)

9. IW Selesnick, The double-density dual-tree DWT. IEEE Trans. Signal Process. 52(5), 1304-1314 (2004)

10. C-M Pun, M-C Lee, Extraction of shift invariant wavelet features for classification of images with different sizes. IEEE Trans. Pattern Anal. Mach. Intell. 26(9), 1228-1233 (2004)

11. MN Do, M Vetterli, The contourlet transform: an efficient directional multiresolution image representation. IEEE Trans. Image Process. 14(12), 2091-2106 (2005)

12. R Eslami, H Radha, Translation-invariant Contourlet transform and its application to image denoising. IEEE Trans. Image Process. 15(11), 3362-3374 (2006)

13. J Ma, G Plonka, The Curvelet transform. IEEE Signal Process. Mag 27(2), 118-133 (2010)

14. I Daubechies, Ten Lectures on Wavelets. (SIAM, Philadelphia, PA, 1992), pp. 269-271

15. A Cohen, I Daubechies, J-C Feauveau, Biorthogonal bases of compactly supported wavelets. Commun. Pure Appl. Math. 45, 485-560 (1992)

doi:10.1186/1687-5281-2012-14

Cite this article as: Shi and Luo: The biorthogonal wavelets that are redundant-free and nearly shift-insensitive. EURASIP Journal on Image and Video Processing 2012 2012:14.

\section{Acknowledgements \\ This study had been supported by National Natural Science Foundation of China (NSFC) under Grant No. 60972156 and Beijing Natural Science \\ Foundation under Grant No. 4102017.}

Received: 20 June 2012 Accepted: 23 August 2012

Published: 7 September 2012

\section{Submit your manuscript to a SpringerOpen ${ }^{\circ}$ journal and benefit from:}

- Convenient online submission

- Rigorous peer review

- Immediate publication on acceptance

- Open access: articles freely available online

- High visibility within the field

- Retaining the copyright to your article

Submit your next manuscript at $>$ springeropen.com 